\title{
Evaluation of circulating Biochemical and Anti-oxidative Biomarkers in Patients with Beta-thalassemia from Lahore Pakistan
}

\author{
Hafiz Muhammad Arsalan, ${ }^{1}$ Nadeem Abbas, ${ }^{2}$ Saira Aslam, ${ }^{3}$ Nabeela Yasmeen, ${ }^{4}$ Rafayur Rehman, ${ }^{5}$ Nasir Farooq ${ }^{3}$
}

\begin{abstract}
Background: $\beta$-thalassemia, one of the two primary kinds of thalassemia, is a typical hereditary issue. Transformations, influencing the different levels of $\beta$-globin quality articulation causing $\beta$-thalassemia, have been found in an overwhelming number.

Objective: To evaluate the biochemical and anti-oxidative status in patients suffering from Thalassemia.

Methodology: This was a comparative study conducted from June $1^{\text {st }}$ to November $30^{\text {th }}, 2018$. A blood sample of $5 \mathrm{ml}$ of 60 diagnosed Thalassemia patients conveniently selected and 60 healthy individuals were taken from a vein in clotted gel vials from Children Hospital and Jinnah Hospital Lahore. Variables included were, Reduce Glutathione (GSH), Catalase (CAT), Superoxide Dismutase (SOD), Malondialdehyde (MDA), Nitric oxide (NO), Vitamin A, C and $\mathrm{E}, \mathrm{Na}^{+}$and $\mathrm{K}^{+}$, Complete Blood Count $(\mathrm{CBC})$, Hemoglobin $(\mathrm{Hb})$ and serum iron level.

Results: MDA level in Thalassemia patients was elevated $(3.02 \pm 0.45)$ vs controls $(1.29 \pm 0.21)(\mathrm{p}=0.000)$. The level of GSH was decreased $(0.18 \pm 0.14)$ as compared to controls $(6.32 \pm 0.13)$. The CAT level was elevated in patients $(2.64 \pm 0.11)$ than controls $(4.11 \pm 1.02)$. The amount of SOD that was elevated in patients $(5.33 \pm 0.81)$ vs controls (3.21 \pm 1.07$)$. Vitamin E level in patients was $(2.38 \pm 0.59)$ vs controls (4.33 \pm 0.95$)$. Red Blood Cells were also decreased in Thalassemia patients $(18.61 \pm 3.32)$ as compared to controls $(39.8 \pm 7.19)$. Serum Iron level was elevated in patients $(131.33 \pm 2.56)$ vs controls $93.21 \pm 1.11$. $(\mathrm{p}=0.000)$.

Conclusion: The expanding level of MDA demonstrates the high rate of lipid peroxidation (oxidative worry) in thalassemic patients. Lower the level of RBC, hemoglobin, and overloaded iron associated with the pathogenesis of Thalassemia.
\end{abstract}

Keywords: Thalassemia, Hb, Iron, Nitric Oxide, SOD, MDA

Article Citation: Arsalan HM, Abbas N, Aslam S, Yasmeen N, Rehman R, Farooq N. Evaluation of circulating Biochemical and Anti-oxidative Biomarkers in Patients with Beta-thalassemia from Lahore Pakistan. JSZMC 2020;11(2):34-38. DOI: https://doi.org/10.47883/jszmc.v11i2.48

\section{Introduction}

$\beta$-thalassemia, one of the two primary kinds of thalassemia, is a typical hereditary issue. The primary driver of this sort of thalassemia is transformations in one of a greater amount of the $\beta$-globin quality loci, and lessened $\beta$-globin generation is caused by these changes. ${ }^{1}$ Transformations, influencing the different levels of $\beta$-globin quality articulation and causing $\beta$ thalassemia, have been found in an awesome number. As indicated by the investigation, the quantity of these changes, distinguished as of late, is not under 200. Uniform dissemination of these changes can't be said geologically, however they have some particular inceptions around the globe. ${ }^{1}$ The significant reasons for very pervasive thalassemia, extraordinarily in Southeast Asia, are over the top populace measure, high birth rate, and consanguineous relational unions (for the most part in India and Pakistan). In Pakistan, the number of births with thalassemic homozygotes is evaluated 7000 which infers 5-5.65 recurrence of general bearer. $^{2}$ With a specific end goal to keep the hemoglobin levels near ordinary, a standout amongst the most customary and helpful medications is normal blood transfusion. $\beta$-thalassemia patients are dependable, in general, need red platelet (RBC) transfusions as it diminishes extramedullary hematopoiesis and development and advancement. ${ }^{3}$ Private division and NGOs assume a key part in the foundation of blood transfusion focuses in Pakistan. As indicated by the reports, the quantity of blood transfusion focuses in the nation is more than 1800 and transfusion administrations are conceivable due

1. Faculty of Allied Health Sciences, Minhaj University Lahore, Pakistan.

2. Department of Biochemistry, University College of Medicine, University of Lahore, Pakistan.

3. School of Biochemistry and MLT, Faculty of Allied Health Sciences, Minhaj University Lahore, Pakistan

4. Department of Physiology, Sheikh Zayad Medical College, Rahim Yaar Khan, Pakistan.

5. Department of Biochemistry, Sheikh Zayad Medical College, Rahim Yaar Khan, Pakistan.

Correspondence: Dr. Hafiz Muhammad Arsalan, Faculty of Allied Health Sciences, Minhaj University Lahore, Pakistan. 
to these divided however quickly expanding focuses. The pervasiveness of this the mellow type of this issue (thalassemia minor) is found in around 10 million populace which constitutes 5$7 \%$ of the populace. Then again, the serious type of this issue (thalassemia major) is observed to be available in 100,000 patients in the nation with an expansion of 5-9 thousand cases each year. Indeed, the patients influenced with thalassemia major generally rely upon the potential treatment of blood transfusion keeping in mind the end goal to expand life expectancy alongside other costly medicinal administrations like chelation treatment. ${ }^{4}$

Related illnesses with this sickness are siderosis, cardiomegaly, jaundice, splenomegaly, significant weakness, and extended bone marrow space. It takes 2-4 months of age for themanifestations to develop and surface. As per the investigation, occasions prompting the oxidative worry in thalassemia are overabundance creation of receptive oxygen intermediates. The life expectancy of erythrocytes might be traded off by this oxidative pressure and a conceivable weighty quickened apoptosis. The nearness of a huge measure of layer bound iron in thalassemic erythrocytes infers the overabundance age of Malondialdehyde (MDA), a result of lipid peroxidation. Press is one of the follow metals that reason unnecessary age of free radical equipped for oxidative harm to erythrocytes. ${ }^{5}$

Extreme hemolytic pallor is initiated by $\beta$ thalassemia and requires standard blood transfusions subsequently. Standard blood transfusions have demonstrated positive outcomes to draw out the life expectancy of the patients, yet auxiliary hemochromatosis has all the earmarks of being a noteworthy test for this situation. While hormonal inadequacy, medullary development, high bone turnover, press gathering, irregular calcium phosphorus, and hypoxia are the diverse variables that add to skeletal illnesses. ${ }^{6}$ The most debilitating life-restricting complexity caused by press over-burden in beta-thalassemia patients is heart malady caused by myocardial siderosis and $71 \%$ of beta-thalassemia patients kick the bucket with these cardiovascular difficulties. Both thalassemia major and intermedia have fringe hemolysis adding to iron deficiency, yet less noticeable in major and more conspicuous in intermedia and happens when layer harm is caused to the fringe erythrocytes by insoluble alpha globin chains. Serious yet inadequate development of the bone marrow (up to 25-30 times typical) is delivered by erythropoietin which is animated by iron deficiency. ${ }^{6}$ The objective of present study was to evaluate the biochemical and Anti-oxidative status in patients suffering from Thalassemia.

\section{Methodology}

This was a comparative study, conducted from June 1 st to November 30th, 2018. Source of Data: $5.0 \mathrm{ml}$ blood sample of 60 diagnosed beta-thalassemia patients and 60 healthy individuals was taken from a vein in clotted gel vials from Mayo hospital and Jinnah Hospital, Lahore. Detailed patient's history, clinical complications, particular smoking, and tobacco chewing were collected from subjects of the study, by providing them a questionnaire. Clinical analysis of the patient was also being taken into consideration. Inclusion criteria: Patient should be Thalassemic and having age from 2-10 years. Exclusion criteria: Individuals having age above 10 were excluded from the present study.

The parameters were estimated, Reduce Glutathione (GSH), Catalase (CAT), Superoxide Dismutase (SOD), Malondialdehyde (MDA), Estimation of Nitric Oxide (NO), Estimation of micronutrients (Vitamin A, Vitamin C, and Vitamin E), and Electrolytes concentration by flame photometer $(\mathrm{Na}+$ and $\mathrm{K}+)$ were estimated. Estimation of Superoxide Dismutase (SOD) was measured and estimated by spectrophotometric method. Determination of Thiobarbituric Acid Reactive Substances (TBARS) in Tissues was done by the spectrophotometric method. ${ }^{8}$ Estimation of Catalase (CAT) was measured by the spectrophotometric method of Aebi. ${ }^{9}$ Determination of GSH was determined by the process of Moron. ${ }^{10}$ Determination of Nitric Oxide (NO) was done by a well-known method such as colorimetric Griess assay. ${ }^{11}$ Estimation of Vitamin C (VIT C) was analyzed by the method described by Roe and Keuther (1943) by spectrophotometrically. ${ }^{12}$ Estimation of Vitamin A (VIT A) was estimated in the plant samples by the Emmutir-Engel reaction as reported by Rosenberg. ${ }^{13}$ Ethical approval was sought from ethical committee. Statistical analysis was done by using Statistical Package for Social Sciences (SPSS) version 20. 


\section{Results}

Data presented in Table I shows the level of micronutrients in Thalassemia patients. The level of vitamin-A in thalassemia patients was recorded as 0.12 and in control persons, the vitamin-A value was 7.14. Vitamin-A value was decreased in thalassemia patients as compared to the control person while statistically significant $(\mathrm{p}=0.000)$. The level of vitamin- $\mathrm{C}$ value was 0.17 in thalassemic patients and in the control person was 6.17 . The vitamin-C value was also decreased as compared to the value of control persons. The value of vitamin-E was noticed in thalassemic patients as 2.38 and the control person as 4.33. The level of vitamin-E was decreased in thalassemic patients as compared to the control person and statistically significant $(\mathrm{p}=0.00)$.

Table-I: Values of different variables compared among Thalassemia patients and Controls.

\begin{tabular}{|l|c|c|c|}
\hline \multirow{2}{*}{ Variables } & $\begin{array}{c}\text { Control } \\
(\mathbf{n}=\mathbf{6 0})\end{array}$ & $\begin{array}{c}\text { Subjects } \\
(\mathbf{n}=\mathbf{6 0})\end{array}$ & $\begin{array}{c}\text { P- } \\
\text { value }\end{array}$ \\
\cline { 2 - 4 } & Mean \pm S.D & Mean \pm S.D & \\
\hline Vitamin A & $7.14 \pm 0.43$ & $0.12 \pm 0.059$ & 0.00 \\
\hline Vitamin C & $6.17 \pm 1.01$ & $0.17 \pm 0.052$ & 0.00 \\
\hline Vitamin E & $4.33 \pm 0.95$ & $2.38 \pm 0.59$ & 0.00 \\
\hline MDA & $1.29 \pm 0.21$ & $3.02 \pm 0.45$ & 0.00 \\
\hline GSH & $6.32 \pm 0.13$ & $0.18 \pm 0.14$ & 0.00 \\
\hline Catalase & $4.11 \pm 1.02$ & $2.64 \pm 0.11$ & 0.00 \\
\hline SOD & $3.21 \pm 1.07$ & $5.33 \pm 0.81$ & 0.00 \\
\hline AGES & $3.33 \pm 0.41$ & $0.14 \pm 0.19$ & 0.00 \\
\hline AOPP & $4.02 \pm 1.03$ & $7.13 \pm 2.17$ & 0.00 \\
\hline Nitric Oxide & $15.21 \pm 2.01$ & $19.21 \pm 0.09$ & 0.00 \\
\hline Sodium (Na+) & $139.11 \pm 9.15$ & $149 \pm 7.06$ & 0.01 \\
\hline Potassium (K+) & $5.37 \pm 1.11$ & $8.01 \pm 2.06$ & 0.03 \\
\hline RBC & $39.8 \pm 7.19$ & $18.61 \pm 3.32$ & 0.000 \\
\hline Hemoglobin & $11.67 \pm 2.29$ & $5.61 \pm 1.76$ & 0.000 \\
\hline Iron & $93 \pm 1.11$ & $131.33 \pm 2.56$ & 0.000 \\
\hline
\end{tabular}

Table-II: Pearson's Correlation between Different Biomarkers.

\begin{tabular}{|c|c|l|}
\hline Parameter & Correlation (r) & P. Value \\
\hline MDA Vs CAT & 0.549 & 0.000 \\
\hline MDA Vs VIT E & -0.611 & 0.000 \\
\hline GSH Vs VIT C & 0.669 & 0.000 \\
\hline GSH Vs AGES & 0.872 & 0.000 \\
\hline CAT Vs VIT E & -0.871 & 0.000 \\
\hline NO Vs VIT C & -0.344 & 0.046 \\
\hline NO Vs VIT E & 0.370 & 0.031 \\
\hline VIT C Vs AGES & 0.612 & 0.000 \\
\hline
\end{tabular}

The level of MDA found in thalassemia patients was 3.02 and in control person was 1.29. So we noticed that the value of MDA was increased in thalassemic patients than control persons and statistically significant $(p=0.000)$. The level of GSH was observed in thalassemic patients as 0.18 and in healthy individuals as 6.32 . We noticed that the value of GSH was decreased in thalassemia patients as compared to the control person. The value of catalase in thalassemia patients was evaluated as 2.64 and in control person was 4.11. We observed that the level of catalase was decreased in thalassemia patients when we compared it with control persons. The resultant value of SOD showed the value was 5.33 in thalassemia patients and in control persons was 3.21. These results told that the value of SOD was increased in patients as compared to control persons while statistically significant $(\mathrm{p}=0.000)$.

The level of AGES was noticed in thalassemic patients as 0.14 and in control persons as 3.33 values show that the level of AGES was decreased when we relate it with control persons. The value of AOPP was recorded in thalassemic patients as 7.13 and in control persons as 4.02. Results predicted that the value of AOPP was increased as compared to control persons. The observed value of nitric oxide in thalassemic patients was 19.21 and in control person was 15.21 told about that the level of nitric oxide was increased than control person while statistically significant $(\mathrm{p}=0.000)$. According to data in table I, Serum Sodium level in thalassemic patients was 149 while in healthy individuals was 139.11 while serum Potassium level observed as 8.01 and in healthy persons was 5.37. Data revealed that serum electrolyte level was elevated in thalassemic patients and statistically significant, $\mathrm{p}=0.01$ and 0.03 , respectively.

The report of CBC profile showed that the values of RBC were 18.61 in thalassemic patients and in control person was 39.8 it reflects that the value of $\mathrm{RBC}$ was decreased as compared to a control person. The recorded value of hemoglobin in thalassemic patients was 5.61 and in control persons 11.67 results showed that the level of hemoglobin was decreased in thalassemic patients as compared to control person. The observed value of iron in thalassemic patients was 131.33 and in control person was 93 showing that the level of iron was increased remarkably in the thalassemic patient as compared to control persons. Table-II shows the correlation of different variables among study subjects. 


\section{Discussion}

$\beta$-thalassemia is an unsettling influence in the hemoglobin chain in which persistent is require constant transfusion of blood to spare their life. Consistent transfusion of blood upgrades the odds of transmission of infection. Transmission of confusion in hemoglobin starting with one individual then onto the next is a noteworthy issue in patients because of insufficient inappropriate data about the disease. ${ }^{11}$ Thalassemia is a sickness that is exchanged by hereditarily from one age to the next ones. It is an aggravated blend of $\alpha, \beta, \gamma, \delta$ chain of hemoglobin. These disordered tends to expand the level of shaky items as precipitation which delivered hemolysis. ${ }^{12}$ These encourage the reason for oxidative harm of cell film. Thalassemia is likewise a reason of haemolytic anaemia. The generation of anomalous RBC drives the splenomegaly. Chelation treatment with multiples times' prompts alternate infections like interminable iron over-burden, cardiovascular maladies, influences the development of body, and affects the sexual improvement of the influenced person. Similar to the current study, it has already been found that oxidative stress increases in patients excess iron. This is the result of full torture of highly active $\mathrm{Fe}^{2+}$ ions causing Fenton and Haber-Weiss reactions and ROS generation. ${ }^{14}$ They started a free autocatalytic process radical lipid peroxidation creates many types of strong genetic breakdown products, including alkoxyl radicals, peroxyl radicals, and aldehydes, i.e. malonedialdehyde. ${ }^{15}$ TBARS rate in $\beta$-thalassemia under investigation adult patients was raised more than $100 \%$. This study result also favored that the collection of LPO products is an appropriate, appropriate marker clear measurement of oxidative stress in clinical manifestations of the disease. ${ }^{16}$ The antioxidant capacity is a result of the full effect of water solubility antioxidants, lipid-soluble antioxidants, and antioxidant enzymes such as superoxide confusion. Oxidative stress is caused by long-term imbalance and antioxidant depression and hyper production of ROS. ${ }^{17}$ The function of the SOD is a direct method of registration of the main ROS content $\mathrm{O}_{2}$ radicals.

There is ample evidence that $\mathrm{RBCs}$, in addition to their functioning as $\mathrm{O}_{2} / \mathrm{CO}_{2}$ carriers and $\mathrm{pH}$ control systems many non-painting works. ${ }^{18}$ This includes a complex role in the implementation of
NO metabolism (through deletion, transfer, release) NO and its metabolites), a systemic redox buffer that contributes to the regulation of systemic redox regulation, as well as transport and releases vasoactive molecules (e.g., ATP and NO / NO metabolites, are defined as erythrocrine activity). ${ }^{19}$ Moreover, scientific studies, RBCs can regulate systemic hemodynamics by influencing blood structures and participating in tissue protection and cardiac homeostasis. ${ }^{20}$ A positive relationship existed amongst MDA and catalase $(r=0.549)$, it demonstrates that as MDA level expanded the catalase level likewise expanded. GSH keeps the amassing of oxidized lipid in cells and assumes an urgent part in the detoxification of $\mathrm{H} 2 \mathrm{O} 2$ and in this investigation the common examination of GSH and vitamin $\mathrm{C}$ mirrors the positive relationship in which both are expanded or diminished collectively (0.699). The connection between MDA and vitamin $\mathrm{E}$ assessed is negative since when the level of MDA is expanded then the level of vitamin $\mathrm{E}$ is diminished $(-0.611)$.

Thalassemic patients indicated upgraded generation of nitric oxide (NO) than in controls. NO is a gobetween of fiery responses. A larger amount of NO reason the diminishing of the sum in vitamin $\mathrm{C}$ since they have a negative connection between each other $(-0.344)$. The level of iron is seen in thalassemic patients is expanded than control which demonstrates that the level of iron in the blood is because of less creation of other parts that is the reason the press level is expanded and prompts different infections. The level of AGES is expanded under the weight of GSH and tight clamp versa. They have a positive relationship $(\mathrm{r}=0.872)$.

\section{Conclusion}

As per the examination of thalassemia patients assessed that this transfusion-transmitted sickness demonstrates the lower the level of RBC, hemoglobin, and other basic mixes when contrast and sound patients. This study demonstrates the serum MDA and potassium are expanded as the contrast with solid kids additionally found that the level of vitamins (A, E, C) is diminished in the assemblage of thalassemic understanding. The expanding level of MDA demonstrates the high rate of lipid peroxidation (oxidative worry) in thalassemic patients. Consequences of present investigation have likewise suggested that more oxidative pressure prompts the cardiovascular 
pressure, liver dysfunctions development impediment, and influences the sexual exercises in these patients. Likewise, we noted that the level of iron was high in thalassemic patients because of the lopsidedness of the hemoglobin chain. There is have to acquaint the most ideal path with control of the thalassemia infection and open the uncommon blood donation centers for the survival of thalassemic patients.

Authors Contribution: HMA: Conception, Drafting and Revising. NA: Conception of work and Design of Work. SA: Acquisition \& analysis. NY: Analysis of data and drafting. RR: Drafting and Revising. NF: Drafting and Revising.

All the authors gave final approval for publication and agreed to be accountable for all aspect of work.

\section{Conflict of Interest: None Sources of Funding: Self}

\section{References}

1. Weatherall DJ. The Thalassemias: Disorders of Globine Synthesis. Hematology. 2006. McGrow Hill 7:633-66.

2. Rizwan AK, Anwar M, Usman W, Asad MJ, Abbasi A, Zaheer HA. Epidemiology of transfusion-transmitted infection among patients with beta-thalassemia major in Pakistan. Jour of Blood Trans, 2016: 2:1-5.

3. Saud AM, Al-Azzawie HF, Al-kazaz AA. Molecular and Biochemical Study on $\beta$-Thalassemia Patients in Iraq. Current Research in Microbiology and Biotechnology 2013: 1(4): 160-165

4. Munir B, Tahira I, Amer J, and Faqir M. Effect of betathalassemia on Hematological and biochemical profiles of female patients. Pak J life Soc Sci 2013:11: 25-28.

5. Filiz S, Ozturk G, Kemahli S, Erbas D, Hasanoglu A. Oxidant and antioxidant status in beta-thalassemia major patients. Medical Sciences 2005:58: 34-38.

6. Laila MF, Abdel-Salam SM, Kamal NM, safy OE, Almalky MAA, Azab S. Nutritional biomarker in children and adolescent with beta thalassemia major, Bio Med Research International 2014: 10: 1-5.
7. Kakkar P, Das B, Viswanathan PN. A modified spectrophotometric assay of superoxide dismutase. Indian J Biochem Biophys 1984:21(2): 130-132.

8. Okhawa H, Ohishi N, Yagi K. Assay for lipid peroxides in animal tissues by thiobarbituric acid reaction. J Anal Biochem 1979: 95: 351-358.

9. Aebi H. Catalase in vitro. Methods Enzymol 1984; 105: 121-6.

10. Moron MS, Depierre JW, Mannervik B. Levels of glutathione, glutathione reductase, and glutathione Stransferase activities in rat lung and liver. Biochim Biophys Acta 1979: 582(1): 67-78.

11. Moshage H, Kok B, Huizenga JR, Jansen PL. Nitrite and nitrate determinations in plasma: a critical evaluation. Clin Chem 1995: 6(1): 892-6.

12. Roe JH, and Keuther CA (1943). The determination of Ascorbicacis in whole blood and urine through the 2-4 Dinitrophenylhydrazine derivative of Dehydroascorbic acid. J Biol. Chem 1943:147: 399.

13. Rosenberg KR. The evolution of modern human childbirth. Physical Anthropology 1992: 35: 89-124.

14. Cighetti G, Duca L, Bortone L, Sala S, Nava I, Fiorelli G, et al. Oxidative status and malondialdehyde in betathalassemia patients, Eur J Clin Invest; 32: (Suppl 1) : S55-60.

15. Chanarat M. Role of lipid peroxidation and antioxidants in aging processes thalassemia. Kitasato Arc Exp Med 1992:65(4): 245-249.

16. Britton RS, Leicester KL, Bacon BR. Iron toxicity and chelation therapy. Int J Hematol 2002:76(3): 219-28.

17. Dhawan V, Kumar KhR, Marwaha RK, Ganguly NK. Antioxidant status in children with homozygous thalassemia. Indian Pediatr 2005;42(11): 1141-1145.

18. Cortese-Krott MM, Rodriguez-Mateos A, Sansone R, Kuhnle GG, Thasian-Sivarajah S, Krenz T, et al. Human red blood cells at work: identification and visualization of erythrocytic eNOS activity in health and disease. Blood 2012; 120: 4229-4237.

19. Gorressen S, Stern M, van de Sandt AM, Cortese-Krott MM, Ohlig J, Rassaf T, et al. Circulating NOS3 modulates left ventricular remodeling following reperfused myocardial infarction. PLoS One 2015; 10: e0120961.

20. Horn P, Cortese-Krott MM, Keymel S, Kumara I, Burghoff $\mathrm{S}$, Schrader J, et al. Nitric oxide influences red blood cell velocity independently of changes in the vascular tone. Free Radic Res, 2011; 45: 653-661. 